\title{
Features wetting and anisotropy of interfacial energy in a metal particle-silicon system
}

\author{
Ludmila P. Aref eva ${ }^{1, *}$, Andrey $V$. Blinov $^{2}$, Alexander $A . \mathrm{Kravtsov}^{2}$, Irina $G$. Shebzukhova ${ }^{3}$ \\ and Alexander $V$. Serov ${ }^{2}$ \\ ${ }^{1}$ Don State Technical University, 344000 Rostov-on-Don, Russia \\ ${ }^{2}$ North-Caucuses Federal University, 355009 Stavropol, Russia \\ ${ }^{3}$ Kabardino-Balkarian State University, 360004 Nalchik, Russia
}

\begin{abstract}
In this paper, we propose a technique for estimating the wetting and anisotropy of the interfacial energy at the interface stabilized by organic compounds of metal particles (cobalt, copper) and semiconductor substrate based on the construction of the Winterbottom equilibrium shape of the crystal. The effective contact angle and the ratio of interphase energies at the particle-substrate interface are calculated from the experimental data of the geometric characteristics of the particles obtained by analyzing the AFM images of the samples. The accuracy of the evaluation of the effective contact angle for all systems is not less than 0.5 $\circ$. It was found that the phenomenon of incomplete wetting is observed in the systems under study. The work of adhesion in the systems under consideration is evaluated. With an increase in the effective contact angle, the ratio of the area of contact to $\mathrm{V}^{2 / 3}$ (V-volume of the particle) in different directions and the work of adhesion is nonlinear. The obtained dependences are qualitatively consistent with thermodynamic states and known data for other systems
\end{abstract}

\section{Introduction}

The production of composite materials and the study of their properties is a priority for the development of materials science [1]. However, experimental studies of the phenomena of wetting and anisotropy of interfacial energy (IE) at the solid-phase interface are few $[2,3]$. The interphase energy of the boundary in the solid system is a fundamental parameter in wetting studies and is used in the Dupre-Young equation to evaluate the thermodynamic work of solid surface adhesion.

In this paper we used the following samples to study the anisotropy of the IE and the wetting characteristics in the particle-substrate system: cobalt particles obtained by the sol drying method and stabilized with ethanediol (Sample 1), cobalt particles (Sample 2) and copper (Sample 3) application to a rotating support and stabilized cetyltrimethylammonium bromide (CTAB). $\mathrm{Si}(100)$ was used as the substrate. The synthesis features, chemical composition and granulometric analysis of stable cobalt and copper sols are described in [4,5].

\footnotetext{
* Corresponding author: Ludmilochka529@mail.ru
} 


\section{Method for determination of interfacial energy and wetting in a solid system based on the construction of Winterbottom}

The Young equation, which is the basis of experimental methods for determining the surface tension of a liquid, is not applicable for determining the contact angle of equilibrium crystals, since it does not take into account the anisotropy of the crystal and the substrate. To determine the contact angle and the equilibrium shape of the crystals on a flat substrate, the Winterbottom method use, described in detail in [6]. The Winterbottom approach is based on a geometric analysis of Wulff's construction of the equilibrium shape of a crystal on a flat solid surface at constant temperature, volume and chemical potential. The case of an equilibrium crystal at $\theta_{e f}<90^{\circ}$ is shown in Fig. 1.

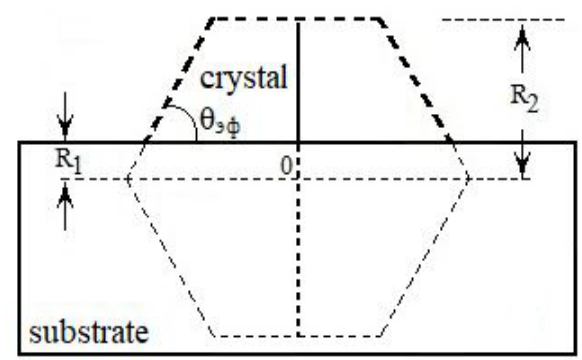

Fig. 1. Scheme of analysis of the Winterbottom of an equilibrium particle on a substrate having an effective contact angle $\theta_{e f}<90^{\circ}$

The IE ratio can be determined from the measurement of two characteristic crystal lengths (Figure 1): the distance from the Wulff point to the interphase boundary $R_{l}$ and from the Wulff point to the upper face $R_{2}$. If $\theta_{e f}>90^{\circ}$, the Wulff point is above the interface of solid phases. If $\theta_{e f}<90^{\circ}$, then the Wulff point is outside the crystal, below the interface of solid phases. Measuring the values $R_{1}$ and $R_{2}$ can be used for description of wetting features in a solid system.

Assuming that there is no mutual phase dissolution in the system or it can be neglected, and there is no change in the real shape of the crystal in comparison with the Wulff model, the ratio $R_{1} / R_{2}$ can be written as:

$$
\frac{R_{1}}{R_{2}}=-\left(\frac{f_{\omega 12}-f_{\omega 13}}{f_{\omega 23}}\right) .
$$

Here $f_{\omega 13}$ - the IE of the substrate at the boundary with the gaseous medium, $f_{\omega 23}-$ the IE particle at the boundary with the gaseous medium, $f_{\omega 12}-$ the IE of the contact boundary of the solid phases. Equation (1) in the case of an isotropic crystal is transformed into Young's equation. Thus, $\arccos R_{1} / R_{2}$ is the effective contact angle $\theta_{\text {ef }}$ of the faceted crystal on the substrate.

We have developed a method for estimating the wetting and anisotropy of IE in a crystal-substrate system using atomic force microscopy (AFM). The data was processed using Nova_Px software. Stages of work:

1) Preparation of metal-containing particles stabilized by organic compounds on the oriented substrate.

2) Obtaining AFM 2D and 3D surface images of samples. 
3) Analysis of sample profiles by the method of Section Analysis (the shape of the particles and their mutual arrangement).

4) Based on the results of the Section Analysis method, we choose of the relative reference level of geometric characteristics and the model of particles on the surface of the substrate for the application of the Grain Analysis method.

5) Application of the Grain Analysis method to identify free-standing particles or their agglomerations, to obtain geometric characteristics of particles. When processing the obtained data, it is necessary to exclude the particles located in the zones of localization of the scanning artifacts or partially extend beyond the scanning area.

6) Determination of characteristic distances $R_{1}$ and $R_{2}$. Calculation of the effective contact angle $\theta_{e f}$ and anisotropy of the IE at the crystal-substrate interface from the obtained geometric characteristics of the crystals.

7) Statistical processing of measurement and calculation results, their graphical representation and analysis.

According to the geometric characteristics of the crystals (the sizes of the section along the $X$ and $Y$ axes (XSize, YSize) and the area of the section (Area) at the zero relative level, the height from the local zero level (Local MaxZ), volume (Volume)), using simple relationships, we can calculate $R_{I}$ and $R_{2}$ and establish the dependence of the effective edge angle and the ratio of IE in the crystal-substrate system on the contact area of the phases.

Depending on the chosen 3-5 stages of the particle, the following approaches can be used to estimate the ratio of the IE and the effective contact angle:

a) The equilibrium shape of the particle tends to the spheroidal segment. The distance from the Wulff point to the upper edge

$$
R_{2 x}=\frac{1}{2 h}\left(\frac{a_{x}^{2}}{4}+h^{2}\right) \quad \text { and } \quad R_{2 y}=\frac{1}{2 h}\left(\frac{a_{y}^{2}}{4}+h^{2}\right),
$$

here $h$ - the height of the particle, $a_{x}$ and $a_{y}$-the length of the particle in the selected direction.

The distance from the Wulff point to the interphase boundary

$$
R_{1 x}=R_{2 x}-h \quad \text { and } \quad R_{1 y}=R_{2 y}-h .
$$

b) The equilibrium shape of the particle tends to an ellipsoidal segment. Using the Point Tool function of the Nova_Px software, the spatial coordinates of two points on the particle surface belonging to the selected direction are set. One of the points must correspond to the top of the particle. Further, substituting in the equation of the ellipse the value of the coordinates of the points, we obtain an expression for the semiaxis of the ellipse, that is, the distance from the top of the particle to the Wulff point. The distance from the Wulff point to the interphase boundary is calculated from (3). The distance from the Wulff point to the upper face in the directions of the $X$ and $Y$ axes of the AFM image

$$
R_{2 x}=\sqrt{\frac{x_{1}^{2} z_{2}^{2}-x_{2}^{2} z_{1}^{2}}{x_{1}^{2}-x_{2}^{2}}} \quad \text { and } \quad R_{2 y}=\sqrt{\frac{y_{1}^{2} z_{2}^{2}-y_{2}^{2} z_{1}^{2}}{y_{1}^{2}-y_{2}^{2}}} .
$$

The work of adhesion (free energy of adhesion with the opposite sign) is expressed through IE at the interfaces. 


\section{Wetting and anisotropy of interfacial energy in the system "stabilized metal particle - silicon (100)"}

The studies were carried out in accordance with the steps described above. Studies of the surface morphology of the obtained samples of cobalt and copper particles by the AFM method ("NT-MDT Ntegra Aura") in contact mode were carried out in [5, 6]. As a result of using the Section Analysis method, it is established that the particles have a clearly defined orientation along the $X$ axis of the samples. Separate particles and agglomerations of particles (Sample 1) are separated from one another at a considerable distance and have dimensions of the order of $\mu \mathrm{m}$. Unlike Sample 1, there are separately standing particles on the surface of Samples 2 and 3 that do not form agglomeration, the distance between the particles is small, much less than $0.1 \mu \mathrm{m}$, and there are practically no regions containing no particles. However, the particles have a long extension in the $X$ and $Y$ directions, of the order of hundreds of $\mathrm{nm}$, and a small height (tens of $\mathrm{nm}$ ). The vast majority of particles (Samples 1, 2 and 3) have one local maximum and do not stick together with neighboring ones. That is, the thin films of cobalt and copper are the island films. The relative level of the horizontal section of particles is chosen like local zero level.

Analysis of all geometric characteristics allows one to conclude that the shape of cobalt particles (Sample 1) tends to a vertically elongated half-ellipsoid, the shape of the cobalt and copper (Samples 2,3) particles to the spheroidal sector.

The effective contact angle of the cobalt particles (Sample 1) was determined in the $X$ and $Y$ directions with respect to the coordinate system of the AFM image of the samples by formulas (1), (3) and (4). The evaluation of both samples 2 and 3 was carried out according to (1), (2), (3). Calculations showed that for most particles the Wulff point is located below the boundary of the particle-substrate contact.

Histograms of the distribution of metal particles of samples 1-3 in terms of the effective contact angle $\theta_{e f}^{x}$ and $\theta_{e f}^{y}$ is shown in Fig. 2. Maximum $\left(\theta_{e f_{\max }}^{x}, \theta_{e f_{\max }}^{y}\right)$ and averaged $\left(\overline{\theta_{e f}^{x}}, \overline{\theta_{e f}^{y}}\right)$ values of effective contact angles in the $X$ and $Y$ directions, the corresponding values $\left(R_{1} / R_{2}\right)_{x}$ and $\left(R_{1} / R_{2}\right)_{y}$ of them and the number of particles are given in Table 1 .

For Sample 1 in the $X$ direction, the values of the effective contact angle lie in the interval from $6^{\circ}$ to $110^{\circ}$, with $74.2 \%$ of the particles have $\theta_{e f}^{x}$ from $22^{\circ}$ to $55^{\circ}$. In the $Y$ direction, the values lie in the interval from $4^{\circ}$ to $108^{\circ}$, with $76 \%$ of the particles have the effective contact angle from $20^{\circ}$ to $50^{\circ}$. For most cobalt particles $\theta_{e f}^{x}>\theta_{e f}^{y}$, and with increasing values, the value $\theta_{e f}^{x} / \theta_{e f}^{y}$ practically does not change. For Sample 2 in the $X$ direction, the values $\theta_{e f}^{x}$ lie in the interval from $6^{\circ}$ to $48^{\circ}$, with $90.7 \%$ of the particles have $\theta_{e f}^{x}$ from $11^{\circ}$ to $30^{\circ}$. In the $Y$ direction, the values $\theta_{e f}^{y}$ lie in the interval from $8^{\circ}$ to $50^{\circ}$, with $87.1 \%$ of the particles have the effective contact angle from $12^{\circ}$ to $29^{\circ}$. For most cobalt particles $\theta_{e f}^{x}<\theta_{e f}^{y}$, and with increasing values $\theta_{e f}^{y}$, the value $\theta_{e f}^{x} / \theta_{e f}^{y}$ decreases. For Sample 3 in the $\mathrm{X}$ direction the values of the effective contact angle lie in the range from $1^{\circ}$ to $30^{\circ}$, with $97.9 \%$ of the particles have the effective contact angle from $2^{\circ}$ to $12^{\circ}$. In the $\mathrm{Y}$ direction, the values $\theta_{e f}^{y}$ lie in the interval from $4^{\circ}$ to $31^{\circ}$, with $96 \%$ of the particles have $\theta_{e f}^{y}$ from $5^{\circ}$ to $16^{\circ}$. For most particles $\theta_{e f}^{x}<\theta_{e f}^{y}$ and as the values $\theta_{e f}^{y}$ increase, the value $\theta_{e f}^{x} / \theta_{e f}^{y}$ decreases. An inverse relationship is observed for the IE ratio. 
Table 1. Effective contact angles and ratios of interfacial energies of samples

\begin{tabular}{|c|c|c|c|c|c|c|c|c|c|}
\hline Sample & $\begin{array}{c}\text { General } \\
\text { number of } \\
\text { particles }\end{array}$ & $\theta_{e f_{\text {max }}}^{x}$ & $\begin{array}{c}\text { Number of } \\
\text { particles }\end{array}$ & $\theta_{e f_{\text {max }}}^{y}$ & $\begin{array}{c}\text { Number of } \\
\text { particles }\end{array}$ & $\overline{\theta_{e f}^{x}}$ & $\overline{\theta_{e f}^{y}}$ & $\left(R_{1} / R_{2}\right)_{x}$ & $\left(R_{1} / R_{2}\right)_{y}$ \\
\hline 1 & 1368 & $35^{\circ}$ & 49 & $33^{\circ}$ & 60 & 43,57 & 37,64 & 0,7245328 & 0,7918635 \\
\hline 2 & 536 & $18^{\circ}$ & 51 & $20^{\circ}$ & 44 & 19,90 & 21,50 & 0,9402881 & 0,9304176 \\
\hline 3 & 1411 & $5^{\circ}$ & 274 & $8^{\circ}$ & 220 & 6,05 & 10,15 & 0,9944303 & 0,9843498 \\
\hline
\end{tabular}
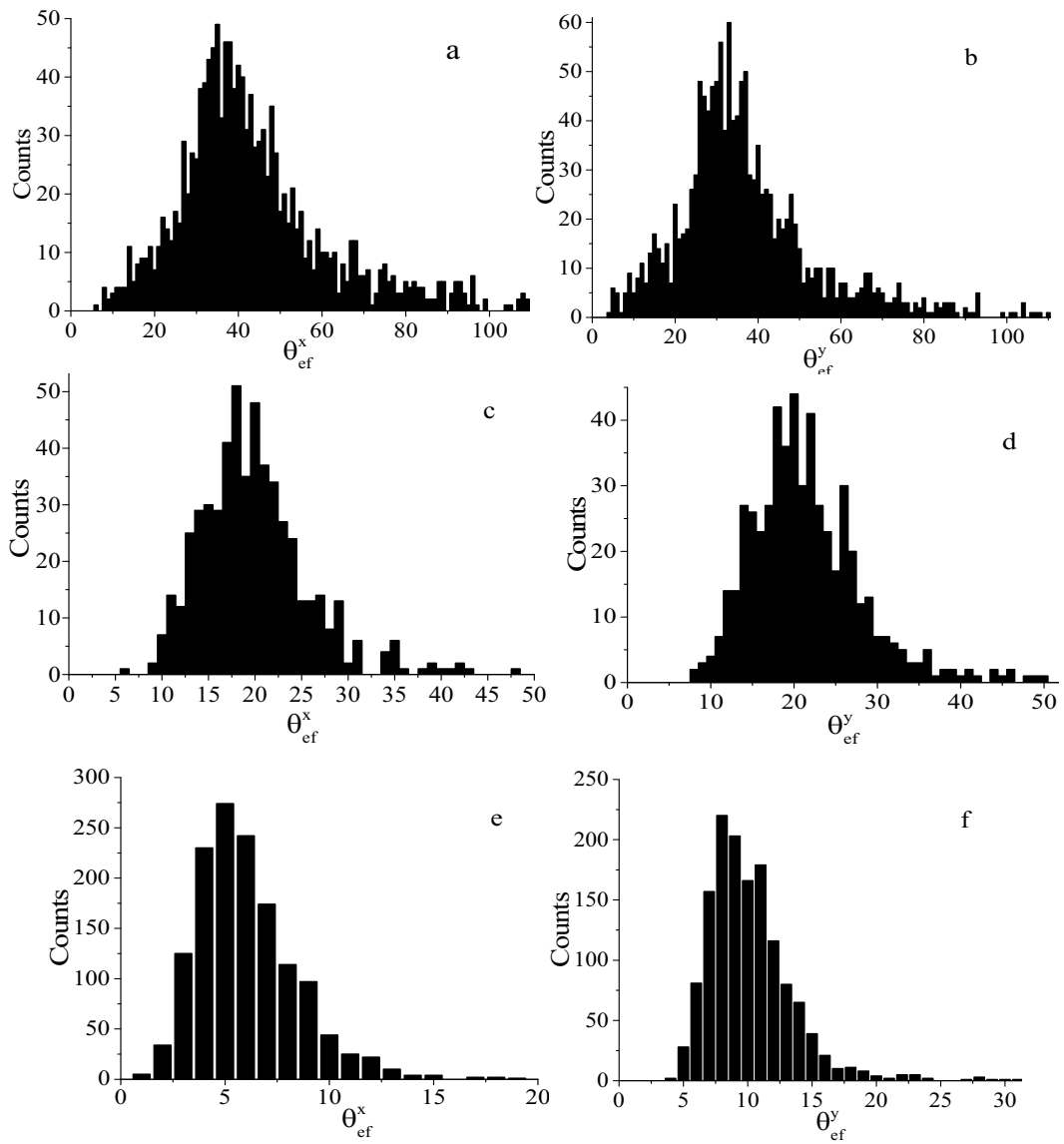

Fig. 2. The histogram of the distribution of metallic particles on the $\mathrm{Si}(100)$ plate in terms of the effective contact angle: $a$-Sample 1 in the $X$-axis direction; $b$-Sample 1 in the $Y$-axis direction; $c$-Sample 2 in the $X$-axis direction; $d$-Sample 2 in the $Y$-axis direction; $e$-Sample 3 in the $X$-axis direction; $f$-Sample 3 in the $Y$-axis direction.

The value of the ratio of the contact area $A$ to $V^{2 / 3}$ ( $V$ is the particle's volume) makes it possible to characterize the wetting phenomenon. The dependence $A / V^{2 / 3}$ on the effective contact angle and taking into account the confidence intervals of the values for samples 1-3 is shown in Fig. 3. The spread of the values $A / V^{2 / 3}$ for Sample 1 in the interval corresponding to the step $1^{\circ}$ is large. The error in estimating the values $A / V^{2 / 3}$ of Samples 2 and 3 is less than $10 \%$. The error in estimating $\theta_{e f}^{x}$ and $\theta_{e f}^{y}$ ordering tenths of a percent for most points. Exceptions are values $A / V^{2 / 3}, \theta_{e f}^{x}$ и $\theta_{e f}^{y}$ for which there are few values (Fig. 3, circles, stars). 


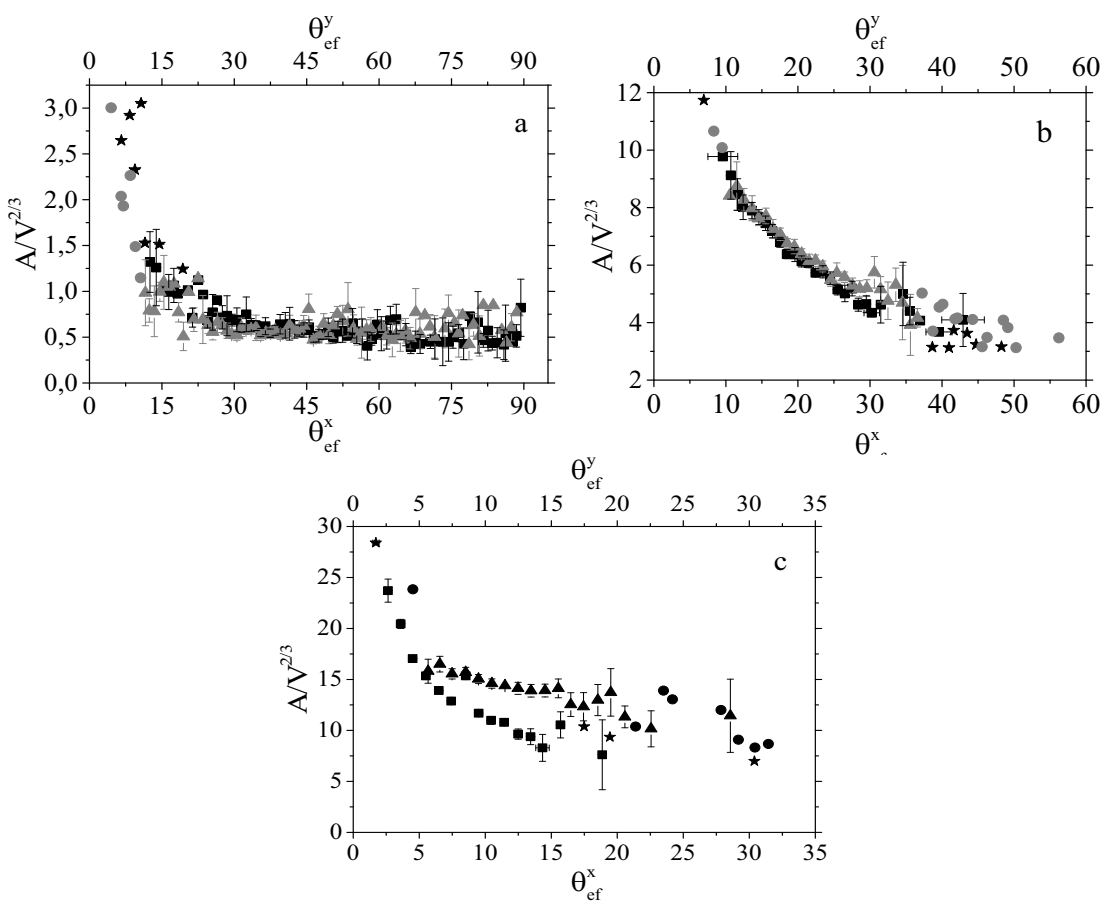

Fig. 3. Dependence of $A / V^{2 / 3}$ of a metal particles stabilized by an organic compound on the effective contact angle: $a$-Sample 1 in the $X$ - (square) and $Y$-axis (grey triangle) directions; $b$-Sample 2 in the $X$ and $Y$-axis directions; $c$ - Sample 3 in the $X$-and $Y$-axis directions

With increasing $\theta_{e f}^{x}$ and $\theta_{e f}^{y}$ the ratio $A / V^{2 / 3}$ decreases nonlinearly. In all samples, the effect of incomplete wetting is observed (Fig. 3). For Sample 1 (Figure 3a), the value $A / V^{2 / 3}$ in the $X$ direction is higher than in the $Y$ direction. For most effective contact angles, the value $A / V^{2 / 3}$ has values from $0.3899 \pm 0.06872$ to $0.77199 \pm 0,19443$ ( $X$ axis) and from $0.502 \pm 0.05671$ to $0.77199 \pm 0.19443$ ( $Y$ axis). In Sample 2, the value $A / V^{2 / 3}$ for the same values of $\theta_{e f}^{x}$ and $\theta_{e f}^{y}$ in the direction of the $X$ axis is smaller than in the direction of the $Y$ axis (Fig. 3b). For most effective contact angles, the value $A / V^{2 / 3}$ has values from $4.40341 \pm 0.47887$ to $9.1216 \pm 0.827$ ( $X$ axis) and from $5.1758 \pm 0.27884$ to $8.7515 \pm$ 0.8392 ( $Y$ axis). In Sample 3, the values $A / V^{2 / 3}$ for the same values of the effective contact angles in the $X$ and $Y$ axis directions practically do not differ (Fig. 3c). For most effective contact angles, the value $A / V^{2 / 3}$ has values from $8.29277 \pm 1.32137$ to $23.71106 \pm 1.13099$ ( $X$ axis) and from $10.15733 \pm 1.76918$ to $16.50215 \pm 0.777189$ ( $Y$ axis).

\section{Conclusion}

From the analysis we can conclude the following:

- at the contact boundary of a metal particle stabilized with an organic compound with an $\mathrm{Si}(100)$ substrate, incomplete wetting is observed, effective contact angles for the systems under consideration are less than $90^{\circ}$;

- anisotropy of the IE and wetting in the directions of the $X$ and $Y$ axes; in the direction of the $X$ axis, wetting is better than in the direction of the $Y$ axis. The greatest anisotropy of the IE and the worst wetting are observed at the contact boundary of the cobalt particles stabilized with ethanediol with the $\operatorname{Si}(100)$ plate; 
- copper particles stabilized by CTAB better wet the oriented silicon plate than cobalt particles in samples 1 and 2;

- the shape of cobalt particles (Sample 1) tends to a vertically elongated half-ellipsoid. The cohesion energy of the stabilized CTAB of the cobalt particle (Sample 2) and copper (Sample 3) is less than the adhesion energy of the cobalt-silicon and copper-silicon boundaries; the shape of the stabilized cobalt and copper particles tends to the spheroidal sector. With an increase in the effective contact angle the work of adhesion has been nonlinearly increased;

- the proposed model of particles and the methodology for estimating the IE boundary of the contact "particle-substrate" allows to adequately describe the wetting phenomenon in the solid system. The obtained dependences are qualitatively consistent with thermodynamic representations and known experimental data for other systems.

\section{References}

1. Yu.A. Olenin, G.V. Lisichkin, Russian Chemical Reviews, 80, 7 (2011)

2. A.V. Chirkov, V.M. Samsonov, Physical and chemical aspects of the study of clusters, nanostructures and nanomaterials, 9 (2017)

3. B.P. Coman, V.N. Yuzevich, PSS, 54, 7 (2012)

4. L.P. Aref eva, A.A. Kravtsov, A.V. Blinov, S.V. Kharchenko, A.V. Serov, I.E. Soloviev, Herald of the Bauman Moscow State Technical University. Series Natural Sciences, 2(71) (2017)

5. L.P. Aref"eva, A.A. Kravtsov, A.V. Blinov, M.S. Suvorov, A.V. Serov, Materialovedenie, 6 (2017)

6. W. D. Kaplan, D. Chatain, P. Wynblatt, W. C. Carter, J. Mater Sci, 48 (2013) 